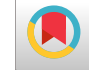

\title{
Factors Affecting the Number of Citations to Clinical Therapeutic Articles Mentioning Level of Evidence
}

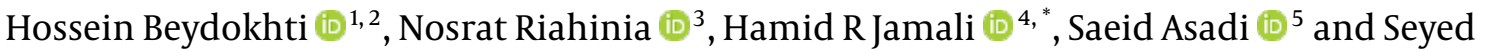 \\ Mohammad Riahi (iD ${ }^{6}$ \\ ${ }^{1}$ Faculty of Medicine, Birjand University of Medical Sciences, Birjand, Iran \\ ${ }^{2} \mathrm{Ph}$. D. Candidate in Knowledge and Information Science, Department of Knowledge and Information Science, Kharazmi University, Tehran, Iran \\ ${ }^{3}$ Department of Knowledge and Information Science, Kharazmi University, Tehran, Iran \\ ${ }^{4}$ School of Information Studies, Charles Sturt University, Wagga Wagga, Australia \\ ${ }^{5}$ Department of Information Science, Faculty of Humanities, Shahed University, Persian Gulf Freeway, Tehran, Iran \\ ${ }^{6}$ Department of Epidemiology and Biostatistics, School of Health, Birjand University of Medical Sciences, Birjand, Iran \\ "Corresponding author: School of Information Studies, Charles Sturt University, Locked Bag 588, Wagga Wagga, NSW 2678, Australia. Email: h.jamali@gmail.com
}

Received 2020 January 25; Revised 2020 May 26; Accepted 2020 May 31.

\begin{abstract}
Background: Level of evidence (LoE) is a hierarchical system for classifying the quality of studies.

Objectives: This study examined the factors affecting the number of citations to clinical articles related to the treatment of human diseases that have included the LoE in their abstracts.

Methods: A total of 3,683 therapeutic articles published between 2011 and 2013 that mentioned the LoE in their abstract and were indexed in PubMed and Web of Science were retrieved. The LoE and type of study design were extracted from abstracts and other bibliographic and citation information was obtained from PubMed and Web of Science databases. Independent samples t-test, oneway ANOVA, Pearson correlation test and linear regression were used to analyze the relationship between the variables.

Results: Articles with level I evidence had the lowest frequency $(290,7.9 \%)$ and articles with level IV had the highest frequency $(1,831$, $49.7 \%$ ). Five-year citations ranged from zero to 215 , with a median of 13 citations. The median values of five-year citations from level I to level $V$ were $20.5,15,14,11$, and 6 citations, respectively. Evaluation of the models to examine the factors affecting the number of citations showed that the change of evidence-level from level I to $\mathrm{V}$ reduced the number of citations $(\mathrm{P}<0.001)$.

Conclusions: Journal Impact Factor, LoE, number of references, number of authors, number of title words, number of pages, article type and subject category accounted for about $25 \%$ of the variation in five-year citations of clinical papers. Clinical papers with high LoE (levels I \& II) received more citations over a five-year period than those with lower LoE (levels III \& IV).
\end{abstract}

Keywords: Citation Analysis, Study Design, Level of Evidence, Evidence-Based Medicine, Clinical Articles, Scientometrics

\section{Background}

Evidence-based medicine with clear methodology guides clinical practitioners on how to access scientific evidence, make it available, and ultimately make the most appropriate decision for treatment. In addition, the number of citations is increasingly being used as performance indicators in research policy and research evaluation systems. Research has shown that the impact of clinical research on decision making by specialists on the patient's bedside is directly related to the type and design of the study. Accordingly, the pyramid of evidence is based on the type and quality of the study method. A well-designed randomized controlled clinical trial (RCT) that can confirm the research hypothesis has long been recognized as the strongest method and technique for gathering valid evidence on the effects of therapeutic interventions (1). According to the Levels of Evidence (LoE) by Oxford Centre for Evidence-based Medicine, medical studies are divided into five levels based on the areas of treatment, diagnosis, prognosis and health economics. The highest level of evidence in the field of therapy, prevention, aetiology and harm is related to randomized clinical trials and their systematic reviews and meta-analysis. At the second level, there are cohort studies. The third level relates to case-control studies and their systematic review and the fourth level relates to case-series and poor quality cohort and case-control studies. Finally, the fifth level includes the expert opinion without an explicit critical appraisal or based on physiology, bench research (2).

Much of the new information reaches the scientific community through the publication of research findings

Copyright (C) 2020, Modern Care Journal. This is an open-access article distributed under the terms of the Creative Commons Attribution-NonCommercial 4.0 International License (http://creativecommons.org/licenses/by-nc/4.0/) which permits copy and redistribute the material just in noncommercial usages, provided the original work is properly cited. 
in journals. The impact of a published article on scientists and experts can be accessed on the basis of its use (3). The number of citations of an article over a given period of time may indicate the importance of its findings by active members of the medical community (4). Whether or not to cite research findings based on study characteristics and whether their results are positive or negative is called citation bias (5). Understanding the citation bias in articles is important because this type of bias can influence the dissemination of information in primary and secondary literature and the attitude of the medical community. In some studies examining the citation of scientific articles in the medical and health sciences, higher levels of evidence have been associated with positive citation bias (4, 6-8). Jamshidi Orak et al. examined the level of evidence of theses and dissertations of nursing dissertations of Iran University of Medical Sciences from 1991 to 2010. Their study showed that $60.7 \%$ of Master's theses and $92.6 \%$ of doctoral theses were in the lowest level of evidence (descriptive and qualitative studies) (9).

Giuffrida and Brown examined the relationship between levels of evidence and the number of citations in veterinary articles over a five-year period. They extracted the scientific and non-scientific properties of original research articles published in five clinical veterinary journals in 2004. The papers were ranked based on one of the levels of evidence-based classification schemes and their citations were extracted over a five-year period. Researchers reported no correlation between the number of citations and the levels of evidence or method of conducting the studies. In this study, it was reported that veterinary specialties and the position of a journal can influence the rate of citations (10).

Amiri et al. in a systematic study examined all articles published in the five spinal research journals in 2010 in terms of the relationship between the level of evidence of the articles and the journals' impact factor (JIF). From 1,658 published articles, they categorized 703 clinical papers based on the table of Oxford levels of evidence between levels I and IV. $66.3 \%$ of articles were in the field of treatment, $14.9 \%$ were prognostic, $11.9 \%$ were diagnosed, $5.3 \%$ were differential diagnosis and $1.6 \%$ were economic/decision analysis. $4.7 \%$ of articles were classified as level I, $23.2 \%$ were level II, 12.5\% were level III and 59.6\% were classified as level IV. $71.8 \%$ of treatment articles were related to level IV. There was no significant difference in the number of articles in levels I and II in five spine journals. Researchers reported that journals with a higher JIF had a higher proportion of evidence-level papers I \& II (11).

Lopez et al. investigated the predictors of citation rates in plastic surgery articles over a five-year period. The subject area, the conflict of interest, the number of authors, and the journal influenced the number of citations. The study showed that high levels of evidence or method of study were not correlated with the number of citations (12).

The inclusion of evidence levels in clinical papers helps develop evidence-based medicine and facilitates the selection of appropriate articles for clinical decision making for specialists. In evidence-based medicine, articles with high levels of evidence have less bias and error, and are, therefore, more suitable for clinical decision making and are more commonly used. Studies have reported inconsistent results regarding the impact of the level of evidence and the number of citations to various journal articles or issues.

This study aims to evaluate factors affecting the number of citations to clinical therapeutic articles mentioning the level of evidence, which indexed in PubMed and Web of Science databases.

\section{Methods}

This study is a cross-sectional study that deploys bibliometric techniques. The databases used in this study were PubMed, Web of Science, and the Journal Citation Reports. The searches were made in July 2019. Using the Advanced Search Tool of PubMed Database, a suitable query was formulated to retrieve research articles related to the treatment of human diseases published from 2011 to 2013 that mentioned in their abstract the evidence level (Table 1).

After the retrieval, abstracts were reviewed to select the articles related to treatment that specified in their abstracts the level of evidence. Then a search query was developed to retrieve bibliographic and citation information of the selected articles from the Web of Science database. Level of evidence, number of references, number of authors, number of title words, number of pages, article type, and subject category were extracted from the information fields using formulas in MS Excel. The study design was determined based on the information contained in the abstract and other information sections of the articles. The electronic publication date of the articles was considered from the beginning of 2011 until the end of 2013, and the number of citations received was extracted from the Web of Science database over a five-year period following their publication. JIF and quartile raking of the journals were also extracted from the Journal Citation Reports database based on the year before publication.

The quantitative data of the study were analysed in two parts: descriptive and inferential statistics. The descriptive section included tables and graphs of absolute and relative frequency distribution, mean and standard deviation, median and Interquartile range. Independent samples T-test, 


\begin{tabular}{|c|c|c|}
\hline Search & Query & Items found \\
\hline \#2 & 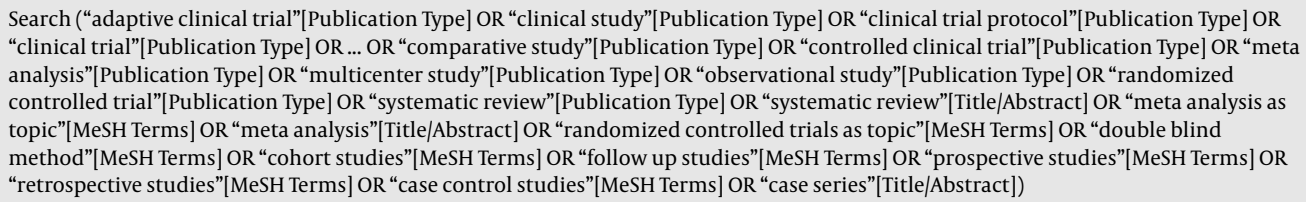 & 4465298 \\
\hline \#3 & Search (\#1 AND \#2) & 2595999 \\
\hline \#4 & Search (\#3) AND (“humans”[MeSH Terms] AND hasabstract[text]) & 2138459 \\
\hline \#5 & Search (\#4)AND (“2011/01/01”[EPDAT] : “2013/12/31”[EPDAT]) & 301745 \\
\hline \#6 & $\begin{array}{l}\text { Search (\#5) AND ("level of evidence"[title/abstract] OR "levels of evidence"[title/abstract] OR "ebm level”[title/abstract] OR "level of } \\
\text { proof"[title/abstract]) }\end{array}$ & 5145 \\
\hline \#7 & Screening of (\#6) & 3904 \\
\hline \#8 & Search (\#7) in Web of Science & 3832 \\
\hline \#9 & Screening of (\#8) & 3683 \\
\hline
\end{tabular}

one-way ANOVA and Pearson correlation test were used to analyse the relationship between variables by SPSS 16 software. Stepwise linear regression analysis was used to determine the factors affecting the number of five-year citations and the effect and contribution of each of the independent variables in explaining the number of five-year citations received was evaluated. VIF index was used to determine the effect of linearity between the independent variables. Adjusted R2 index was used to determine the variance explained by the independent variables. This method also adjusted the effect of the number of variables entered in the regression model. All interpretations are reported in a linear regression model based on a logarithmic criterion. P value $<0.05$ was considered significant.

\section{Results}

Between 2011 and 2013, 3,683 clinical articles on the treatment of human diseases that specified the level of evidence in their abstract were published and indexed in PubMed and Web of Science databases. The number of articles based on the year of electronic publication from 2011 to 2013 respectively was 907 (24.6\%), 1214 (33\%) and 1562 (42.4\%). Descriptive indicators of variables including number of authors, number of title words, number of references, page counts of articles, JIF and 5-year citations count are reported in Table 2.

One article was in French and the rest were in English language. More than 95\% of articles were journal articles $(3,297)$ and conference papers $(234)$. The articles were published in 80 different journals. About 22\% of articles were published in the American Journal of Sports Medicine; and Knee Surgery, Sports Traumatology, Arthroscopy. More than half of the articles were published in top $25 \%$ of journals on each topic and the share of Lippincott Williams \& Wilkins was greater than Elsevier, Springer and other publishers.

A total of 14,237 authors from over 3,000 scientific institutions contributed to the publication of these articles. The University of California with 120 articles, the University of Texas with 117 articles and Harvard University with 111 articles contributed the most. The authors were from 70 countries, with the United States accounting for more than $40 \%(1,485)$ of the articles. There were 14 articles in which Iranian authors contributed.

About $64 \%$ of the articles were orthopedic, according to the first subject group on the Web of Science. Frequency distribution of the articles by the level of evidence showed that about $50 \%$ of the articles are classified in the fourth level of evidence and the average number of five-year citations decreased from high levels of evidence to low levels of evidence (Table 3). Investigating JIF in the year prior to the publication of the article shows that articles with highlevel evidence were published in journals with a higher average JIF.

The results of comparing the mean number of citations received by the articles showed a significant difference between all the subgroups of the variables studied ( $P$ $<0.001)$. The Tukey test for each of the variables showed that the articles were published in 2011 received more citations than in 2012 and 2013. Articles on Obstetrics \& Gynecology received more citations than other subject cat- 


\begin{tabular}{|c|c|c|c|c|c|}
\hline Variables & Mean \pm SD & Median & [Q1-Q3] & Min & $\operatorname{Max}$ \\
\hline Author count & $5.31 \pm 2.27$ & 5 & {$[4-6]$} & 1 & 27 \\
\hline Title words count & $14.15 \pm 4.69$ & 14 & {$[11-17]$} & 2 & 36 \\
\hline References count & $29.77 \pm 13.64$ & 28 & [20 - 37] & 1 & 146 \\
\hline Pages count & $7.44 \pm 2.24$ & 7 & {$[6-9]$} & 2 & 23 \\
\hline IF 2 years & $2.50 \pm 1.08$ & 2.21 & {$[1.57-3.31]$} & 0.30 & 8.25 \\
\hline IF 2 years (2018) & $3.82 \pm 1.63$ & 3.61 & {$[2.37-4.87]$} & 0.48 & 9.80 \\
\hline 5 years citation count & $19.15 \pm 20.00$ & 13 & {$[7-24]$} & 0 & 215 \\
\hline
\end{tabular}

egories. The number of citations decreased from level I to level $\mathrm{V}$, and the articles with high levels of evidence received more citations than those with low levels of evidence. Based on the type of study, systematic review and meta-analysis articles received the most and the casecontrol studies received the lowest number of citations. Also, the articles written by the Japanese corresponding authors were the least and the Italian articles were the most cited. Articles published in Q1 journals received the most citations in a five-year period $(\mathrm{P}<0.001)$.

The results of Pearson correlation coefficient test to calculate the relationship between the variable of the JIF and the number of 5-year citations of articles indicated a positive and significant relationship between these two variables $(\mathrm{r}=0.36, \mathrm{P}<0.001)$. There was also a positive and significant relationship between the number of references and the pages count of articles $(\mathrm{r}=0.53, \mathrm{P}<0.001)$ and between the references count and the number of authors of articles $(\mathrm{r}=0.36, \mathrm{P}<0.01)$. The correlation matrix of variables showed in Table 4.

Stepwise linear regression analysis was used to investigate and determine the factors affecting the number of citations. The variables were entered into the models in order of importance. Variables predictor accounted for about $25 \%$ of the variation in the number of citations of therapeutic articles $(\mathrm{F}=77.04, \mathrm{P}<0.001)$. Factors affecting the number of citations are reported in Table 5 .

Five-year JIF, level of evidence, references count, page count, authors count and other variables that are shown in Table 5 significantly predict the number of citations. For each unit of evidence-level change, from level I to $\mathrm{V}$, the number of citations drops by 0.14 standard deviations. It is also expected that by one unit increase in the score of fiveyear JIF, the number of citations will be increased by 0.35 standard deviation.

\section{Discussion}

Research has shown that the impact of clinical research on the decision making of specialists on the patient's bedside is directly related to the type and design of the study. Accordingly, the system of evidence classification is based on the type and quality of the research method (1). Although the level of evidence is not an absolute criterion for the quality of articles, studies of highlevel evidence are more reproducible and the results derived from the design of such studies will have a more reliable clinical application (13). Moreover, the purpose of medical research is to uncover information that can advance understanding of disease processes and patient care. Physicians often pay attention to the credibility of the journal for reading related articles in their field of expertise. The number of times an article is cited over a given period may indicate the relative importance of its findings by active research members of the medical community. Besides, the amount of citations an article is important for authors, journals, publishers, and readers; and citation is increasingly used as a measure of impact and quality in research policy and evaluation systems. This reported the results of a comprehensive study on the distribution of appropriate clinical papers for evidence-based medical practice in the field of human disease treatment between 2011 and 2013. The 3,683 articles that specified the level of evidence were published in 80 journal titles. Fourteen journals published more than $85 \%$ of these articles.

Usually, the number of citations is affected by the research and its quality (14). Examination of the number of citations received for five-year clinical trials related to disease treatment showed that high-level evidence (level I \& II) received more citations than low-level evidence (level III \& IV). The results of past studies on the relationship between the level of evidence and citations are not conclusive or consistent. While some previous research found no relationship (10,15-18), some other studies found positive relationships between the two $(14,19)$. The inconsistency could be due to different subject areas studied, the definition of 


\begin{tabular}{|c|c|c|c|}
\hline Variables & Frequency $(\%)$ & Citation, Mean \pm SD & PValue \\
\hline Published year & & & $<0.001$ \\
\hline 2011 & $899(24.73)$ & $2.65^{A} \pm 0.95$ & \\
\hline 2012 & $1200(33.01)$ & $2.48^{\mathrm{B}} \pm 0.96$ & \\
\hline 2013 & $1536(42.26)$ & $2.53^{\mathrm{B}} \pm 0.93$ & \\
\hline Subject categories & & & $<0.001$ \\
\hline Orthopedics & $2328(64.04)$ & $2.60^{\mathrm{AB}} \pm 0.94$ & \\
\hline Surgery & $531(14.61)$ & $2.34^{\mathrm{B}} \pm 0.99$ & \\
\hline Obstetrics \& gynecology & $195(5.36)$ & $2.80^{\mathrm{A}} \pm 0.88$ & \\
\hline Critical care medicine & $180(4.95)$ & $2.42^{\mathrm{B}} \pm 0.95$ & \\
\hline Clinical neurology & $151(4.15)$ & $2.57^{\mathrm{AB}} \pm 0.94$ & \\
\hline Urology \& nephrology & $123(3.38)$ & $2.57^{\mathrm{AB}} \pm 0.78$ & \\
\hline Medicine, research \& experimental & $85(2.34)$ & $2.30^{\mathrm{B}} \pm 0.85$ & \\
\hline Others & $42(1.16)$ & $1.88^{\mathrm{C}} \pm 1.01$ & \\
\hline Level of Evidence & & & $<0.001$ \\
\hline I & $288(7.92)$ & $3.01^{A} \pm 0.93$ & \\
\hline II & $618(17.00)$ & $2.72^{\mathrm{B}} \pm 0.94$ & \\
\hline III & $858(23.60)$ & $2.61^{\mathrm{BC}} \pm 0.87$ & \\
\hline IV & $1799(49.49)$ & $2.41^{\mathrm{C}} \pm 0.94$ & \\
\hline $\mathrm{v}$ & $72(1.98)$ & $1.91^{\mathrm{D}} \pm 1.15$ & \\
\hline Document types & & & $<0.001$ \\
\hline Systematic review \& meta-analysis & $210(5.78)$ & $3.13^{\mathrm{A}} \pm 0.78$ & \\
\hline RCT \& clinical trial & $691(19.01)$ & $2.73^{\mathrm{B}} \pm 1.01$ & \\
\hline Cohort study & $531(14.61)$ & $2.67^{\mathrm{BC}} \pm 0.93$ & \\
\hline Case-control study & $161(4.43)$ & $2.45^{\mathrm{D}} \pm 0.92$ & \\
\hline Case series study & $861(23.69)$ & $2.49^{\mathrm{CD}} \pm 0.96$ & \\
\hline Other & $1181(32.49)$ & $2.33^{\mathrm{D}} \pm 0.87$ & \\
\hline Corresponding author address & & & $<0.001$ \\
\hline USA & $1474(40.55)$ & $2.65^{\mathrm{AB}} \pm 0.93$ & \\
\hline South Korea & $264(7.26)$ & $2.46^{\mathrm{BC}} \pm 0.89$ & \\
\hline France & $225(6.19)$ & $2.30^{\mathrm{C}} \pm 0.88$ & \\
\hline China & $168(4.62)$ & $2.26^{\mathrm{C}} \pm 0.98$ & \\
\hline Germany & $162(4.46)$ & $2.71^{\mathrm{B}} \pm 0.78$ & \\
\hline Italy & $142(3.91)$ & $2.90^{A} \pm 0.93$ & \\
\hline Other & $1200(33.01)$ & $2.46^{\mathrm{BC}} \pm 0.98$ & \\
\hline Journals & & & $<0.001$ \\
\hline Am. J. Sports Med. & $412(11.33)$ & $3.24^{\mathrm{A}} \pm 0.81$ & \\
\hline Knee Surg. Sports Traumatol. Arthrosc. & $389(10.70)$ & $2.45^{\mathrm{D}} \pm 0.91$ & \\
\hline Plast. Reconstr. Surg. & $356(9.79)$ & $2.63^{\mathrm{C}} \pm 0.91$ & \\
\hline Arthroscopy & $301(8.28)$ & $2.99^{\mathrm{B}} \pm 0.83$ & \\
\hline Other & $2177(59.89)$ & $2.35^{\mathrm{D}} \pm 0.92$ & \\
\hline Journal quartile & & & $<0.001$ \\
\hline Q1 & $1942(53.43)$ & $2.82^{\mathrm{A}} \pm 0.90$ & \\
\hline Q2 & $857(23.58)$ & $2.35^{\mathrm{B}} \pm 0.91$ & \\
\hline Q3 & $623(17.14)$ & $2.04^{C} \pm 0.85$ & \\
\hline Q4 & $213(5.86)$ & $2.29^{\mathrm{B}} \pm 0.92$ & \\
\hline
\end{tabular}




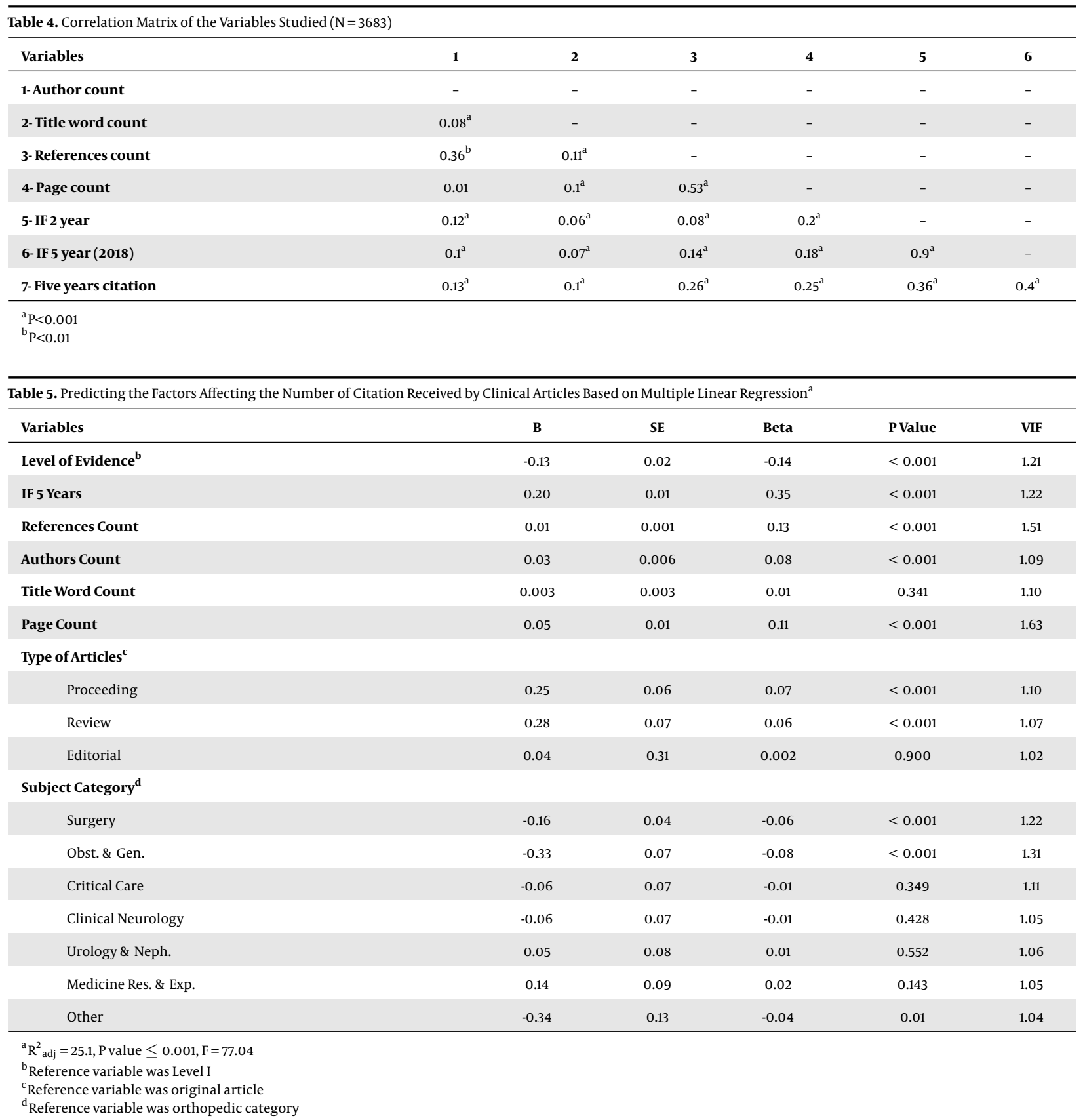

citation impact, and sampling. In our study, we included all levels of evidence, something that was lacking in some past studies.

Systematic reviews and meta-analysis articles received more citations than articles with other types of designs. Also, randomized clinical trials and cohort studies received more citations than case-control and case-series studies. In this research, the type of study design was extracted from the information contained in the abstracts of the articles and retrospective and prospective studies and follow-up studies were considered as other types of study design. Similarly, some past studies have shown that research method and study design and some other factors such as sample size and industrial financial support influence the number of citations $(12,20,21)$

Subject difference in the number of citations received has been shown in past studies (22). Our study showed that the majority of articles with a level of evidence mentioned 
in the abstract were in the orthopedic subject area (64\%), and surgery (14\%). Statistical tests showed a significant difference between the mean numbers of citations for different topics. Articles on Obstetrics \& Gynecology had the highest citations.

The correlation between the different variables was positive. The correlation between the number of five-year citations and JIF, the number of references, article length, number of authors, and number of title words were weak. Multiple linear regression analysis showed that the evidence levels alone accounted for $4.2 \%$ of citation variation and the evidence levels along with the five-year JIF accounted for $18.4 \%$ of the variation in the number of citations received in clinical articles. Also adding other variables and modeling them across the seven models showed that fitting the variables of the levels of evidence, the fiveyear JIF, the number of references, the number of authors, the number of pages, the types of articles, and the subject influenced the number of citations received and explains 25.1\% of these changes (Table 5). Similarly, Antoniou et al. found that topic, study design, paper length could predict citation rate (20). Another study also that factors such as sample size, JIF and study design could influence the citation rate (6).

The findings showed that more than 78 percent of these articles were on orthopedics and surgery. The study showed that the articles on gynecology have received more citation than others.

\subsection{Conclusions}

Number of citations was significantly correlated with JIF, level of evidence, number of references, number of authors, number of title words, length of article, subject, type of study design, geographical area of corresponding author, journal and publisher. Statistical tests predicted that JIF, the levels of evidence, the number of references, and the number of authors were more effective than the other factors on changes in the number of citations received. Clinical papers with high LoE (levels I \& II) received more citations over a five-year period than those with lower LoE (levels III \& IV).

\section{Footnotes}

Authors' Contribution: HB: Conceptualization, Methodology, Formal analysis, Data curation, Writing - original draft. NR: Methodology, Supervision, Writing - review and editing. HRJ: Methodology, Supervision, Writing - review and editing. SA: Methodology, Writing - review and editing. SMR: Formal Analysis, Writing - review and editing.

Conflict of Interests: The authors declare that they have no conflict of interest.
Ethical Approval: This study was approved by the Ethics Committee of the Birjand University of Medical Sciences, Birjand, Iran. (Ethical No.: IR.BUMS.REC.1398.041).

Funding/Support: This work was done at the expense of the authors.

\section{References}

1. Farhadi H. [The role of evidence based medicine in explanation of research articles: systemic evaluation of evidence]. Journal of Dental School. 2006;24(1):135-48. Persian.

2. Phillips B, Ball C, Sackett D, Badenoch D, Straus S, Haynes $\mathrm{B}$, et al. The Oxford Centre for Evidence-based Medicine - Levels of Evidence. 2009, [cited 2018 Sep 11]. Available from: https://www.cebm.net/2009/06/oxford-centre-evidence-basedmedicine-levels-evidence-march-2009/.

3. Callaham M, Wears RL, Weber E. Journal prestige, publication bias, and other characteristics associated with citation of published studies in peer-reviewed journals. JAMA. 2002;287(21):2847-50. doi: 10.1001/jama.287.21.2847. [PubMed: 12038930].

4. Patsopoulos NA, Analatos AA, Ioannidis JP. Relative citation impact of various study designs in the health sciences.JAMA.2005;293(19):23626. doi: 10.1001/jama.293.19.2362. [PubMed:15900006].

5. Higgins J, Green S. Cochrane Handbook for Systematic Reviews of Interventions Version 5.1.0: The Cochrane Collaboration. 2011. Available from: https://handbook-5-1.cochrane.org/index.htm.

6. Bhandari M, Busse J, Devereaux PJ, Montori VM, Swiontkowski M, Tornetta Iii $\mathrm{P}$, et al. Factors associated with citation rates in the orthopedic literature. Can J Surg. 2007;50(2):119-23. [PubMed: 17550715]. [PubMed Central: PMC2384258].

7. Kumar M, Gopalakrishna C, Swaminath PV, Mysore SS. Evidencebased surgery-evidence from survey and citation analysis in orthopaedic surgery. Ann R Coll Surg Engl. 2011;93(2):133-8. doi: 10.1308/003588411X12851639107872. [PubMed: 21073824]. [PubMed Central: PMC3293308].

8. Okike K, Kocher MS, Torpey JL, Nwachukwu BU, Mehlman CT, Bhandari M. Level of evidence and conflict of interest disclosure associated with higher citation rates in orthopedics. J Clin Epidemiol. 2011;64(3):331-8. doi: 10.1016/j.jclinepi.2010.03.019. [PubMed: 20947295].

9. Jamshidi Orak R, Alibeik MR, Banani GH. [A Survey on the Levels of Evidence of Nursing Theses and Dissertations in Type 1 Medical Universities of Iran, 1991-2010]. Iran Journal of Nursing. 2012;25(78):52-61. Persian.

10. Giuffrida MA, Brown DC. Association between article citation rate and level of evidence in the companion animal literature. J Vet Intern Med. 2012;26(2):252-8. doi: 10.1111/j.1939-1676.2011.00869.x. [PubMed: 22268956].

11. Amiri AR, Kanesalingam K, Cro S, Casey AT. Level of evidence of clinical spinal research and its correlation with journal impact factor. Spine J. 2013;13(9):1148-53. doi: 10.1016/j.spinee.2013.05.026. [PubMed: 23806347].

12. Lopez J, Calotta N, Doshi A, Soni A, Milton J, May JJ, et al. Citation Rate Predictors in the Plastic Surgery Literature. J Surg Educ. 2017;74(2):1918. doi:10.1016/j.jsurg.2016.08.005. [PubMed: 27651051].

13. Kay J, Memon M, de Sa D, Duong A, Simunovic N, Ayeni OR. Does the Level of Evidence of Paper Presentations at the Arthroscopy Association of North America Annual Meetings From 2006-2010 Correlate With the 5-Year Publication Rate or the Impact Factor of the Publishing Journal? Arthroscopy. 2017;33(1):12-8. doi: 10.1016/j.arthro.2016.05.032. [PubMed: 27453453].

14. Cheng KL, Dodson TB, Egbert MA, Susarla SM. Which Factors Affect Citation Rates in the Oral and Maxillofacial Surgery Literature? J 
Oral Maxillofac Surg. 2017;75(7):1313-8. doi: 10.1016/j.joms.2017.03.043. [PubMed: 28445696].

15. Ahmad SJS, Ahmed AR, Exadaktylos AK, McWhinnie D, Nickel F, Hakky $\mathrm{SM}$, et al. Systematic review on citation classics in minimally invasive gastrointestinal surgery. J Minim Access Surg. 2018;14(4):265-72. doi: 10.4103/jmas.JMAS_149_18. [PubMed: 30106025]. [PubMed Central: PMC6130179].

16. Sochacki KR, Jack R2, Nauert R, Harris JD. Correlation Between Quality of Evidence and Number of Citations in Top 50 Cited Articles in Rotator Cuff Repair Surgery. Orthop J Sports Med. 2018;6(6):2.3259671187766E+15. doi: 10.1177/2325967118776635. [PubMed: 29977936]. [PubMed Central: PMC6024541].

17. Gambin-Botella J, Ayala M, Alfonso-Beltran J, Barrios C. Predominance of Studies With Poor Level of Evidence Among the Top 100 Most Cited Studies on Idiopathic Scoliosis: A Bibliometric and Content Analysis. Spine Deform. 2018;6(4):373-83. doi: 10.1016/j.jspd.2017.12.003. [PubMed: 29886907].

18. Jack R2, Sochacki KR, Morehouse HA, McCulloch PC, Lintner DM, Harris JD. Correlation Between Quality of Evidence and Number of Citations in Top 50 Cited Articles on Elbow Medial Ulnar Collateral
Ligament Surgery. Orthop J Sports Med. 2018;6(4):2.3259671187682E+15. doi: 10.1177/2325967118768216. [PubMed: 29780841]. [PubMed Central: PMC5954328].

19. Azer SA, Azer S. What can we learn from top-cited articles in inflammatory bowel disease? A bibliometric analysis and assessment of the level of evidence. BMJ Open. 2018;8(7). e021233. doi: 10.1136/bmjopen2017-021233. [PubMed: 30002009]. [PubMed Central: PMC6082456].

20. Antoniou GA, Antoniou SA, Georgakarakos EI, Sfyroeras GS, Georgiadis GS. Bibliometric analysis of factors predicting increased citations in the vascular and endovascular literature. Ann Vasc Surg. 2015;29(2):286-92. doi: 10.1016/j.avsg.2014.09.017. [PubMed: 25462541].

21. Farshad M, Sidler C, Gerber C. Association of scientific and nonscientific factors to citation rates of articles of renowned orthopedic journals. European Orthopaedics and Traumatology. 2013;4(3):125-30. doi: 10.1007/s12570-013-0174-6.

22. Tahamtan I, Safipour Afshar A, Ahamdzadeh K. Factors affecting number of citations: a comprehensive review of the literature. Scientometrics. 2016;107(3):1195-225. doi: 10.1007/s11192-016-1889-2. 